\title{
Design of Lightweight Structural Components for Direct Digital Manufacturing
}

\author{
Xin Ning * and Sergio Pellegrino ${ }^{\dagger}$ \\ California Institute of Technology, Pasadena, CA 91125
}

\begin{abstract}
The rapid growth in direct digital manufacturing technologies has opened the challenge of designing optimal micro-structures for high-performance components. Current topology optimization techniques do not work well for this type of problems and hence in this paper we propose a technique based on an implicit representation of the structural topology. The detailed microstructure is defined by a continuous variable, the size distribution field, defined over the design domain by chosen shape functions. We can optimize the structural topology by optimizing only the weights of the size distribution field and, for any given size distribution, we use standard meshing software to determine the actual detailed microstructure. We have implemented the optimization loop using commercial CAD and FEA software, running under a genetic algorithm in MATLAB. Application this novel technique to the design of a sandwich beam has produced designs that are superior to any standard solid beam or even optimized truss structure.
\end{abstract}

\section{Introduction}

Recent advances in direct digital manufacturing technologies have made it possible to replace conventionally machined load-bearing parts with space-frame-like components. This development opens the challenge of finding optimal architectures for these space frames, in order to maximize the benefits of these new design and manufacturing approaches. Problems of this kind belong to the field of structural topology optimization which has seen major advances in recent years. However, despite the availability of high-performance computers and powerful commercial CAD and FEA software, existing approaches are suitable only for problems in which the micro-structure to be designed is rather coarse. If our objective is to design fine micro-structures then current topology optimization techniques are unable to converge to a solution that is even close to optimal. In this paper we introduce an implicit topology optimization method that successfully addresses these drawbacks.

The paper begins with a brief review of relevant structural topology optimization methods followed by an introduction to direct digital manufacturing technologies for metallic structures. Section III introduces the design problem, a simply-supported beam, that is chosen as a focus of the present study and then the proposed implicit topology optimization method is presented. Section IV describes the optimization algorithm used in this study and the software implementation of the whole optimization cycle is presented in Section V. Section VI presents the results of the optimization problem and Section VII introduces two metrics to compare the performance of beams designed by the present approach with standard beams and a simple truss. Section VIII concludes the paper.

\section{Background}

This section provides a brief review of current approaches to structural topology optimization, followed by an overview of direct digital manufacturing technologies for metallic materials.

\footnotetext{
${ }^{*}$ Graduate Student. Graduate Aerospace Laboratories, California Institute of Technology, 1200 E. California Blvd. MC 105-50.xning@caltech.edu

$\dagger$ Joyce and Kent Kresa Professor of Aeronautics and Professor of Civil Engineering, Graduate Aerospace Laboratories, California Institute of Technology, 1200 E. California Blvd. MC 301-46. AIAA Fellow. sergiop@caltech.edu
} 


\section{A. Approaches to Structural Topology Optimization}

Current approaches to structural topology optimization can be classified into continuum methods and discrete methods. ${ }^{1}$

In the continuum methods, the design domain is discretized into a set of continuum finite elements and the material properties of each element are varied, or an element may even be removed, in order to obtain the optimal material distribution for given loads and boundary conditions. ${ }^{2,3}$ There are many computational implementations of this type of approach, from open source to commercial packages. For example, Bendsøe and Sigmund ${ }^{4}$ provide a compact 99-line MATLAB code for the Solid Isotropic Material with Penalization (SIMP) method and Abaqus/CAE version 6.11 includes an optimization module based on the homogenization method. However, this approach does not generate actual structures and thus extensive post-processing of the optimization results is required to convert them into actual members and voids. An example result obtained from this approach is provided in Figure 1, showing an adaptive wing leading edge that has been optimized for single-point actuation. It can be seen that there are some isolated patches of material and nonsmooth structural boundaries. As a result, a great deal of post-processing would be required to transform this particular solution into a practical structure.

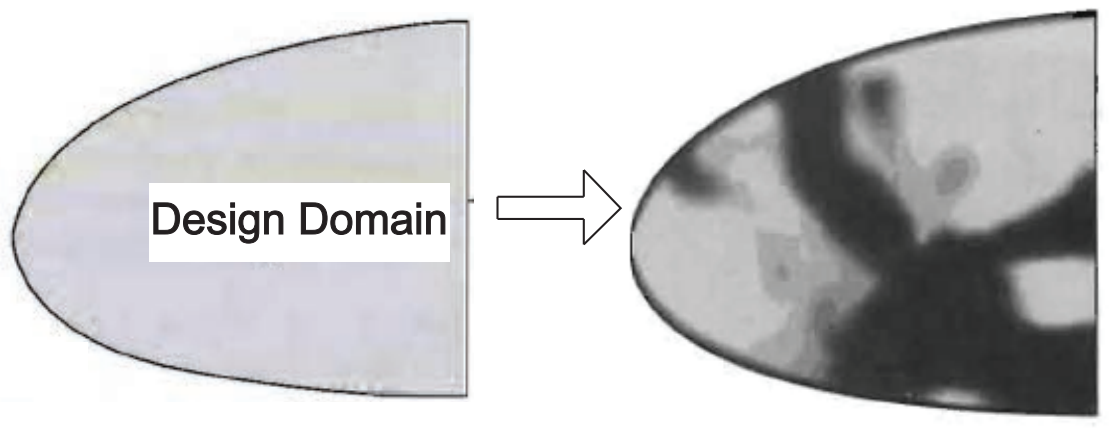

Figure 1: Continuum topology optimization of aircraft leading wing. ${ }^{5}$

Discrete optimization methods have a long history dating back to the 1900's and significant improvements have been made in recent decades. ${ }^{1}$ There are two key approaches to discrete topology optimization: groundstructure based methods and shape-grammar methods. ${ }^{6}$

In ground-structure based methods, the design domain is represented by a ground structure that contains the full set of possible structural components, attached to a grid of nodes that has a high degree of connectivity. By eliminating some of the members included in the ground structure and also varying the cross-sectional dimensions of its members, one can search for structures with optimal topology and member sizes. Although the ground structure method is rather intuitive, it may also create impractical structures with floating components or disconnected load points. An example result obtained from this method is provided in Figure 2 showing some isolated parts.
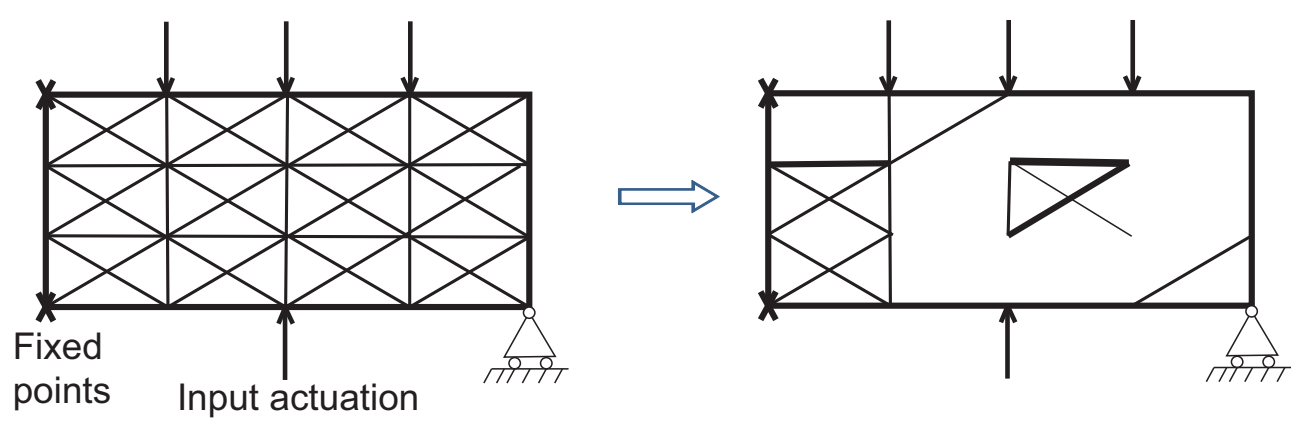

Figure 2: Impractical structure obtained from ground structure based method. ${ }^{8}$

To avoid these difficulties $\mathrm{Lu}$ and $\mathrm{Kota}^{7,8}$ introduced the load-path technique. In this case, a finite set of load paths connecting the points of application of the loads to the support points is predefined and an 
optimization is carried out to select a specific set. Santer and Pellegrino ${ }^{5}$ combined the load-path technique with the K Shortest Simple Path (KSSP) method in which only the shortest K load paths (where K is an integer) are included in the set, instead of all possible load paths. Figure 3 shows an optimized adaptive wing leading edge obtained by the KSSP technique. Load path methods produce fully connected structures. They are still a type of ground-structure method, since they work with a predefined set of structural members. Although load-path methods produce more practical structures than other ground-structure methods, their results are still dependent on the definition of the ground set, which limits their generality.

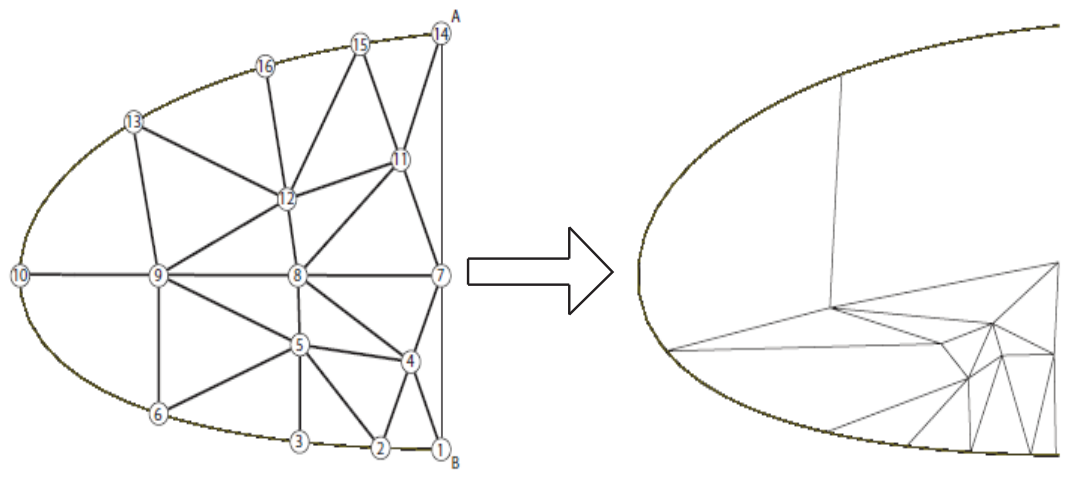

Figure 3: Aircraft adaptive leading edge obtained from KSSP method. ${ }^{5}$

In the shape-grammar method, ${ }^{9,10}$ instead of eliminating structural members originally included in the ground structure, the topology of the structure is grown from an initially defined, simple structure, through repeated applications of a series of rules ("shape grammar"). Simulated annealing is used to apply the shape grammar and optimize the structural topology; Figure 4 shows an example of this approach.

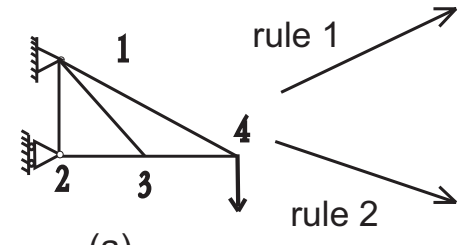

(a)

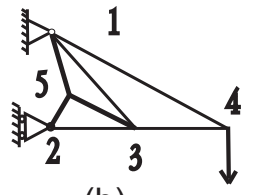

(b)

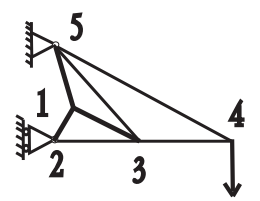

(c)

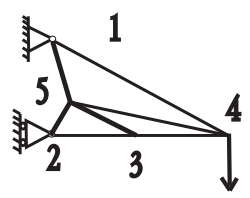

(d)

Figure 4: Example of shape grammar rules. ${ }^{10}$

In discrete approaches the structural topology has to be defined explicitly which, for the type of problems of interest in this paper, introduces a very large design space. For example, 50 load paths in the design domain would lead to a design space containing $2^{50}-1 \approx 1.12 \times 10^{15}$ possible structures. Discrete approaches successfully avoid the drawbacks of continuum approaches, but the large design space that is generated by 
them significantly increases the difficulty of finding optimal topologies.

\section{B. Direct Digital Manufacturing}

Direct Digital Manufacturing (DDM) is an automatic manufacturing process that creates physical parts directly from 3D CAD files or data using computer-controlled additive fabrication techniques. DDM technology can manufacture complex geometries with high energy efficiency, low material waste and fast speed.

Current direct digital manufacturing technologies can produce structural components from a wide range of materials including polymers and metals such as steel, titanium and alloy. Two main DDM technologies, laser sintering and electron beam melting, can be used to manufacture structural components. Laser sintering technology uses a high power laser to fuse small metallic particles by scanning cross-sections generated from 3D CAD files. ${ }^{11,12}$ The CAD files are sliced into layers and the structure is built layer by layer, starting from the bottom. The layout of a typical laser sintering machine is shown in Figure 5.

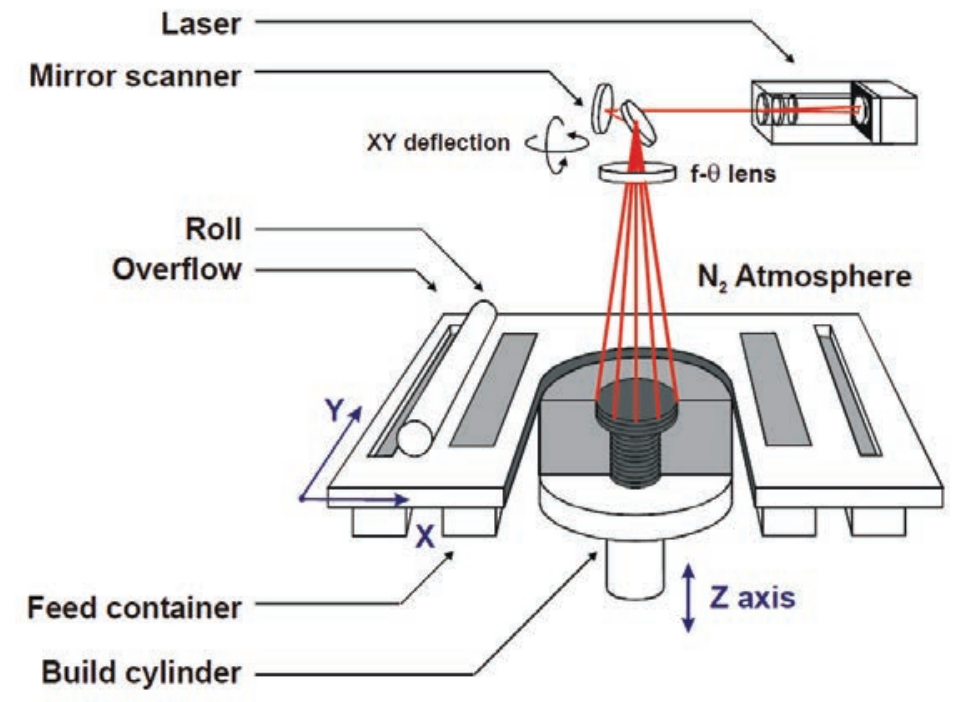

Figure 5: Schematic of laser sintering machine. ${ }^{12}$

In electron beam melting (EBM) technology electrons are emitted from a filament which is heated to $>2500^{\circ} \mathrm{C}$ and are accelerated through the anode to half the speed of light. When the electrons hit the heated powder, kinetic energy is transformed to heat and the metal is melted. ${ }^{13}$ Similar to laser sintering, EBM melts the metallic powder layer by layer, a schematic of EBM is shown in Figure 6. Figure 7 shows a titanium cube with an internal microstructure designed by the present authors and made by CalRam Inc. using Electron Beam Melting.

DDM technology has made it possible to transform designs of almost limitless complexity into reality, and without significant increases in cost. In the rest of this paper we will focus on optimization techniques that can exploit this new capability.

\section{Implicit Topology Optimization}

To overcome the drawbacks of both continuum and discrete approaches, we will adopt an implicit representation of the structural topology. The detailed microstructure is defined by a continuous variable, the size distribution field, defined over the design domain by a weighted interpolation of some chosen shape functions. Thus instead of searching the entire design space, we can optimize the structural topology by optimizing only the weights of the size distribution field. For any given size distribution, we will use standard meshing software to determine the actual detailed micro-structure.

In this section we provide details of our proposed optimization technique. We will first introduce a beam design problem that will be used as an example to present our method. Then, we explain the key idea of the new method and finally its implementation. 


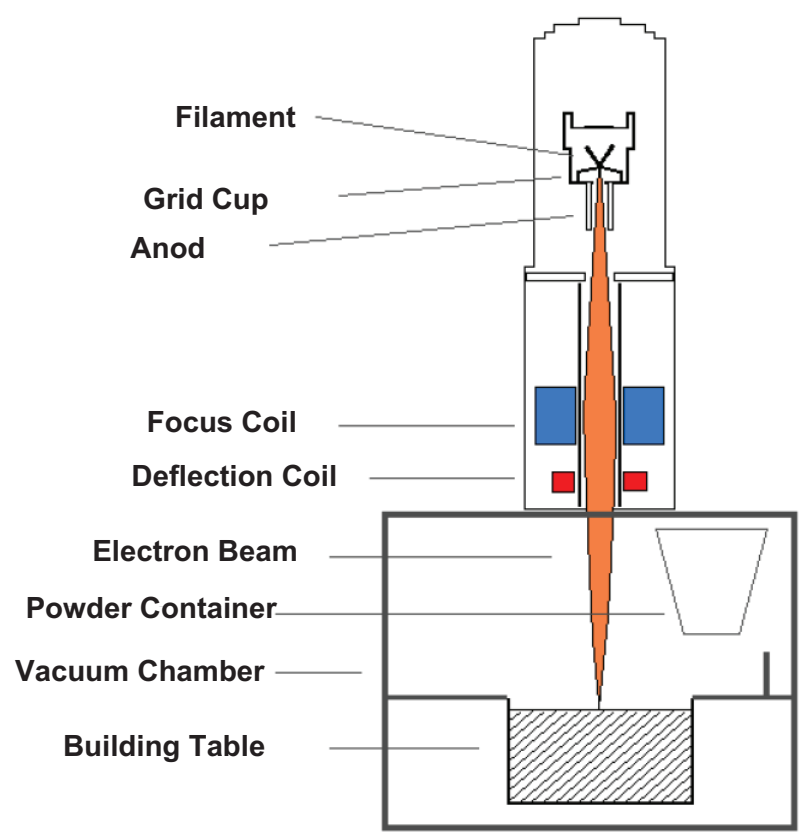

Figure 6: Schematic of EBM technology. ${ }^{13}$

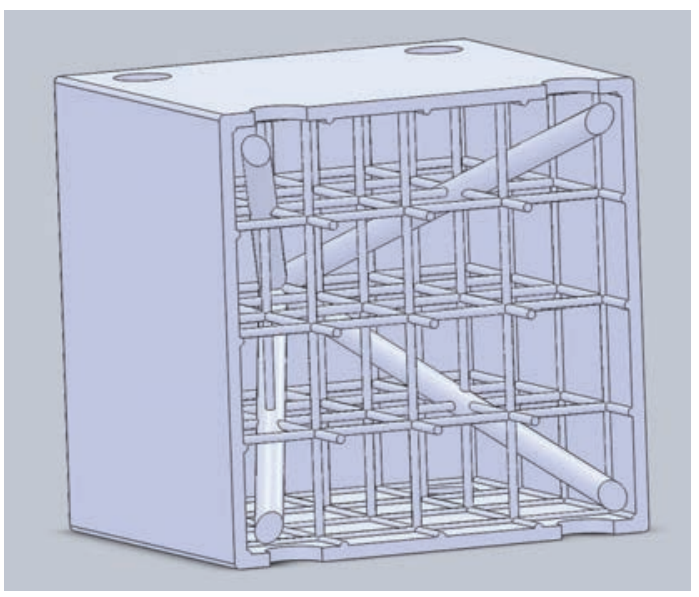

(a)

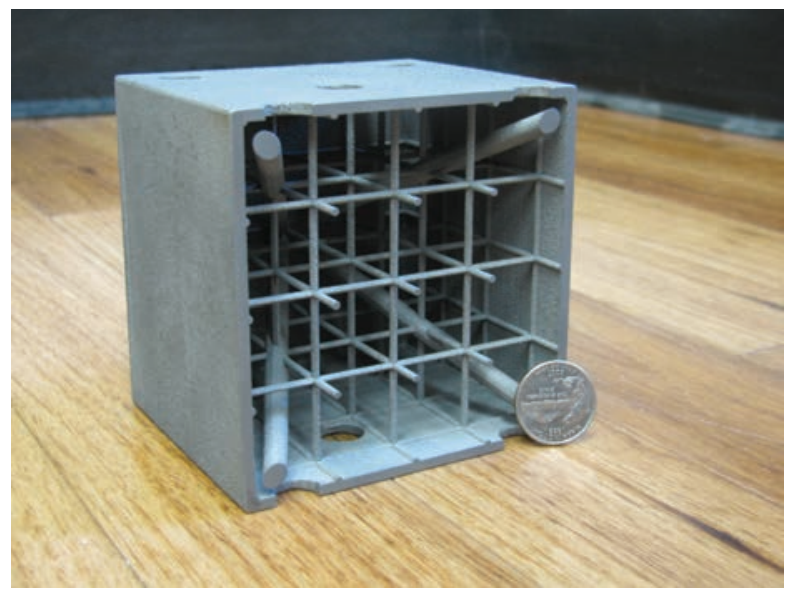

(b)

Figure 7: Lightweight cube made by EBM: (a) cut view generated from CAD file; (b) sectioned titanium cube. 


\section{A. Optimization Problem}

We will study a simply supported 2D beam under uniformly distributed load. Beam design is a classical problem and hence we will be able to compare the results obtained from our study with well-known analytical solutions.

A schematic of the design problem is provided in Figure 8, showing a simply supported beam with length of $1 \mathrm{~m}$ and height of $0.1 \mathrm{~m}$ under a uniformly distributed load of $1000 \mathrm{~N}$. The beam has a continuous outer skin and an internal microstructure. The skin is $2 \mathrm{~mm}$ thick and the microstructure consists of rods with a diameter of $2 \mathrm{~mm}$; initially, only the topology of the microstructure will be varied.

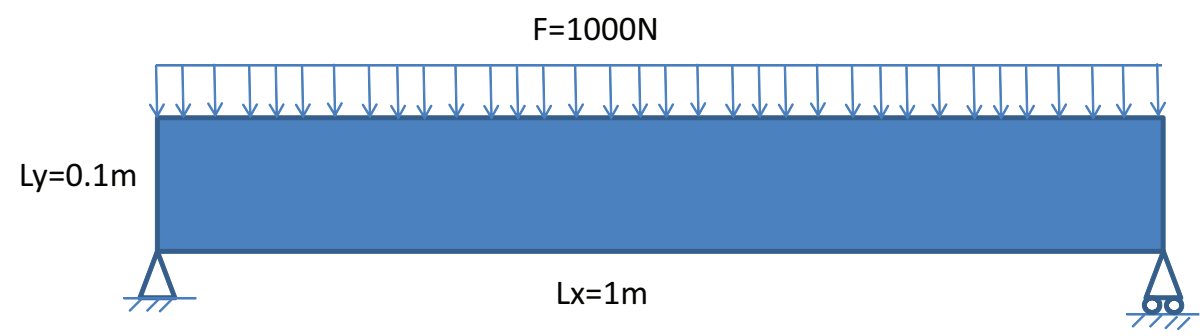

Figure 8: Schematic of beam design problem.

Our objective is to design a lightweight beam that is also very stiff. These two objectives will be combined as a weighted sum to define the following combined objective:

$$
\text { Minimize } f(T)=\omega_{1} \frac{\operatorname{Mass}(T)}{\operatorname{Mass}_{0}}+\omega_{2} \frac{U(T)}{U_{0}}
$$

where $\omega_{1}, \omega_{2}$ are weighting coefficients such that their sum is one, the design variable $T$ and the objective $U$ are the topology of the microstructure and the total strain energy of the beam, respectively.

Since the loading is prescribed, maximizing stiffness is equivalent to minimizing the deflection of the beam and, since the strain energy can be considered a generalized measure of deformation, $U$ is used to characterize structural stiffness. $M a s s_{0}$ and $U_{0}$ are a reference mass and strain energy, respectively, used to normalize the objectives. We will take $\omega_{1}=0.3$ and $\omega_{2}=0.7$ in this problem.

It is possible that a structural design with large deformation but very small mass could produce a small value of the combined objective function. We avoid such structures by introducing a constraint on the maximum deformation. Valid designs will have to satisfy mechanical constraints on stress, to avoid yielding of the material, and buckling, to avoid that any member of the micro-structure buckles. We define the parameter, $\lambda$, as the ratio between the critical buckling load and the applied load:

$$
\lambda=\frac{F_{\max }}{F}
$$

Here $F_{\max }$ is the critical buckling load and $\lambda$ must be larger than 1 if the applied load is to be lower than the critical buckling load. $\lambda$ can be obtained by finding the first eigenvalue through a buckling analysis of a structure under fixed loading $F=1000 \mathrm{~N}$.

In conclusion, the optimization problem can be formulated as follows:

$$
\begin{array}{lll}
\text { Minimize: } & f(T)=\omega_{1} \frac{\operatorname{Mass}(T)}{\operatorname{Mass}_{0}}+\omega_{2} \frac{U(T)}{U_{0}} & \\
\text { subject to: } & \sigma_{Y} \leq \sigma_{0} & \text { (Stress constraint) } \\
& \lambda \geq 1 & \text { (Buckling constraint) } \\
& \delta \leq 4 \mathrm{~mm} & \text { (Deformation constraint) }
\end{array}
$$

where $\sigma_{Y}$ and $\delta$ are the Mises stress and the maximum deformation of the top surface of the beam, respectively. 


\section{B. Structural Size Field}

In an explicit representation of structural topology the positions of the nodes and their connectivity would be defined explicitly. Our proposed approach is to use a continuous function, the structural size field, to describe the variation of the length of the structural members. This idea is similar to adaptive finite element meshing in stress analysis; adaptive meshing techniques focus on the distribution and size of the mesh rather than the actual positions of the nodes or their connectivity. Similarly, in implicit topology optimization we define the size field and then generate a corresponding microstructure, such that its size distribution conforms to the size field.

Two simple examples of analytically defined size fields are provided in Figure 9, showing rectangular domains covered by triangular elements with edge length conforming to $h(x, y)$. The two examples are generated by a simple MATLAB mesh generator, DistMesh, developed by Persson. ${ }^{15}$ These examples show that complex topologies can be obtained without explicit definitions of nodes or connectivity, which shows that optimization of the structural size field is a viable approach to try.

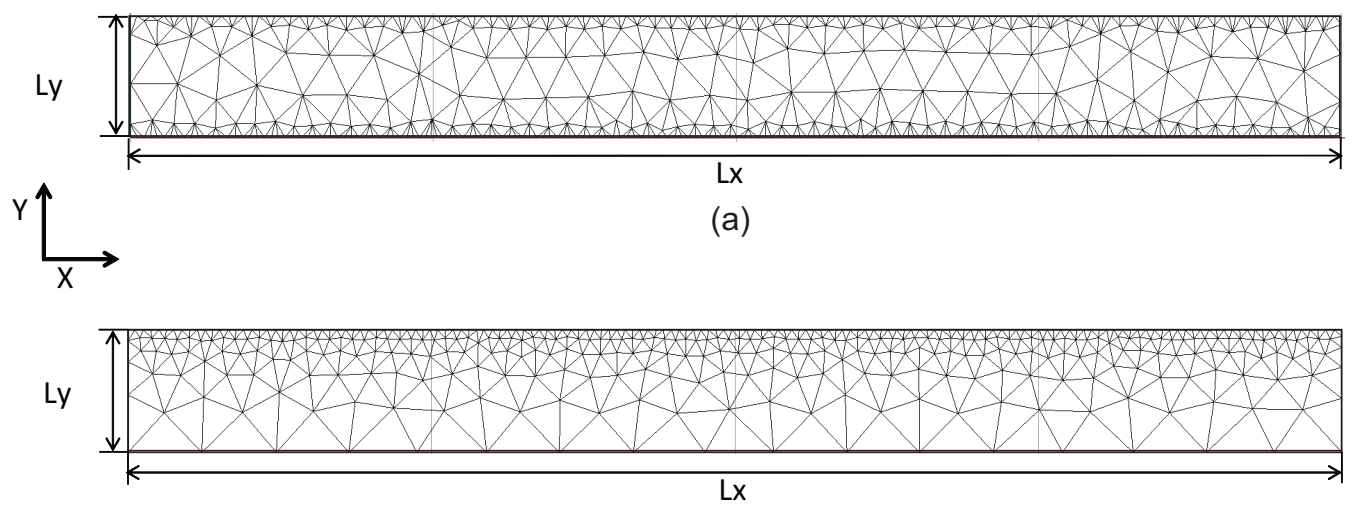

(b)

Figure 9: Microstructures defined by analytical size field: (a) $h(x, y)=h_{0}\left\{1+5 \sin \left(\frac{y}{L_{y}} \pi\right)\right\}$; (b) $h(x, y)=$ $h_{0}\left\{1+7 \frac{L_{y}-y}{L_{y}}\right\}$.

Since using a single analytical function for the structural size field would be a severe restriction, the field will be defined on the nodes of the background mesh shown in Figure 10. There are 36 nodes in this mesh, corresponding to 36 independent variables, $h_{i}(i=1,2, \ldots, 36)$, from which the size field over the whole domain is obtained by linear interpolation of those 36 variables.

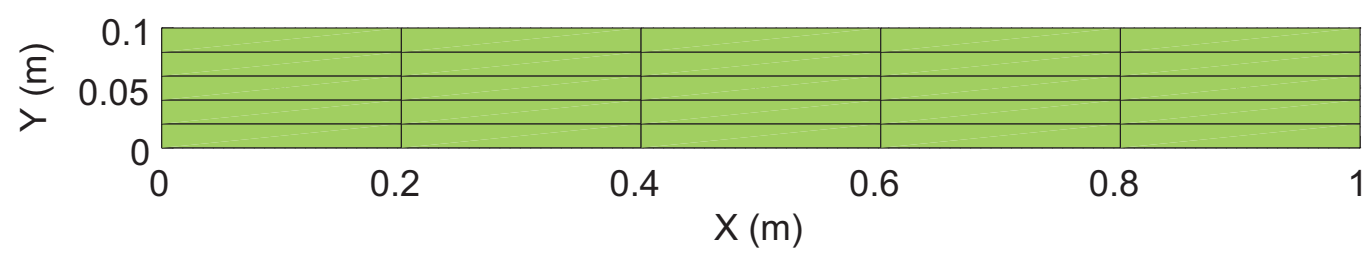

Figure 10: Background mesh.

$$
h(x, y)=\operatorname{Interpolation}(x, y, \vec{h}), \vec{h}=\left(h_{1}, h_{2}, \ldots, h_{36}\right)
$$

Thus, formulating the topology optimization problem in terms of these 36 variables, it becomes: 


$$
\begin{aligned}
& \text { Minimize : } f(\vec{h})=\omega_{1} \frac{\operatorname{Mass}(\vec{h})}{\operatorname{Mass}_{0}}+\omega_{2} \frac{U(\vec{h})}{U_{0}}, \vec{h}=\left(h_{1}, h_{2}, \ldots, h_{36}\right) \\
& \text { subject to: } \sigma_{Y} \leq \sigma_{0} \quad \text { (Stress constraint) } \\
& \lambda \geq 1 \quad \text { (Buckling constraint) } \\
& \delta \leq 4 \mathrm{~mm} \quad \text { (Deformation constraint) } \\
& h 0 \leq h_{i} \leq 6 \cdot h 0, \text { where } h 0=0.0064 \mathrm{~m}
\end{aligned}
$$

\section{Structural Topology Generation}

The structural topology is produced by a mesh generator that takes the structural size field as an input and generates a corresponding microstructure, as shown in Figure 11. Mesh generation methods fall into three main categories: hierarchical spatial decompositions, advancing-front methods, and Delaunay based methods. ${ }^{16}$ Among these methods, Delaunay based methods promise greater flexibility and are suitable for

adaptive meshing. ${ }^{17}$ A mesh generator should (1) be able to generate meshes of high quality; (2) be able to obtain a mesh that conforms to a specified size field; (3) be fast and robust.

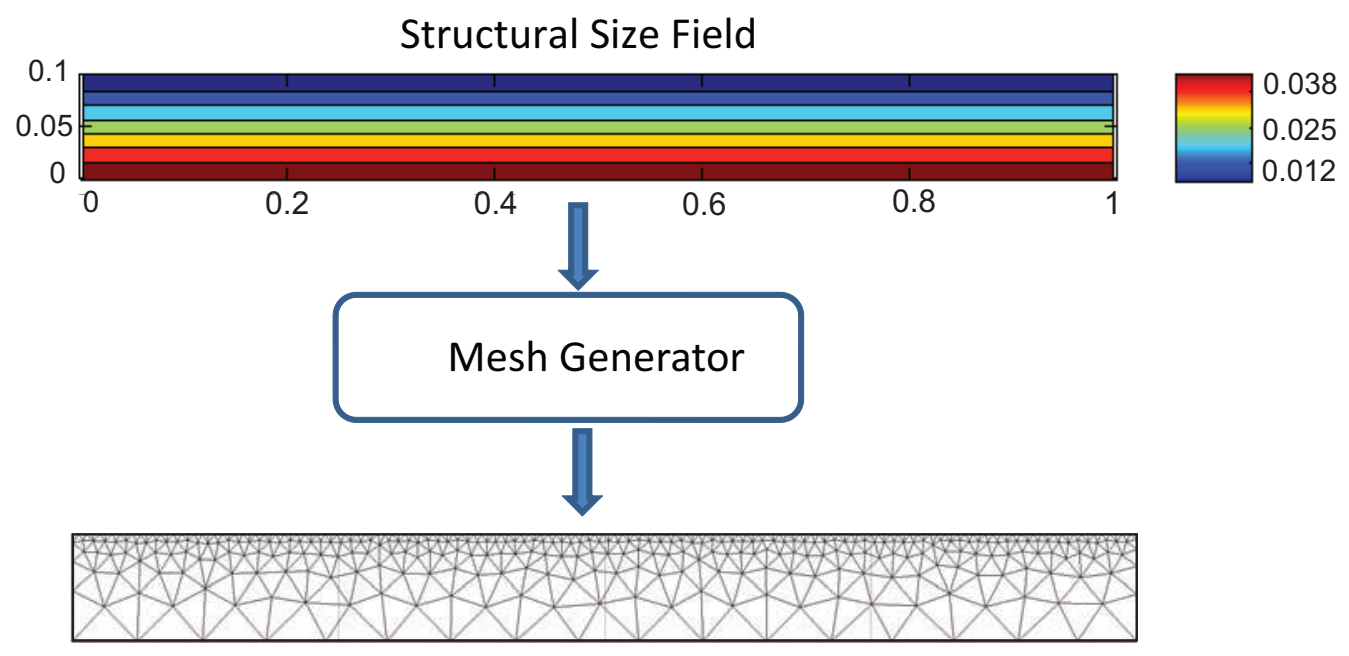

Figure 11: Procedure of structural topology generation

In this paper we use the Delaunay based mesh generator MESH2D developed by Darren Engwirda. MESH2D is a Matlab toolbox of 2D meshing routines that allows for the automatic generation of unstructured triangular meshes for 2D geometry. An iterative algorithm is used that optimizes the mesh topology/nodes position to achieve high quality triangulations. ${ }^{18}$ In each step a constrained Delaunay triangulation is generated and then nodes are added or removed from the mesh until the required element size distribution is satisfied.

\section{Optimization Algorithm}

The convexity of this optimization problem cannot be guaranteed, as it cannot even be described by mathematical equations. In addition, the design space for this problem is hard to analyze. Therefore, we use a genetic algorithm to solve the optimization problem. Genetic algorithms pioneered by Goldberg ${ }^{19}$ have many advantages over other algorithms, including the ability to handle non-convex problems while dealing with different types of domains. ${ }^{6,20}$

The flow chart in Figure 12 shows the general procedure of a genetic algorithm. There are three main steps: selection, crossover and mutation. A population of 20 individuals (structures) is generated. Good individuals are selected as parents according to the fitness of each individual. Crossover combines the parents to create offspring which contains features of the parents. Mutation is then conducted to increase the diversity of the population. The worst parents are replaced by a new generation, but the best individuals are kept. 
This process continues until a prescribed maximum number of generations is reached. An open-source MATLAB genetic algorithm toolbox developed by the University of Sheffield, ${ }^{21}$ is used in this paper.

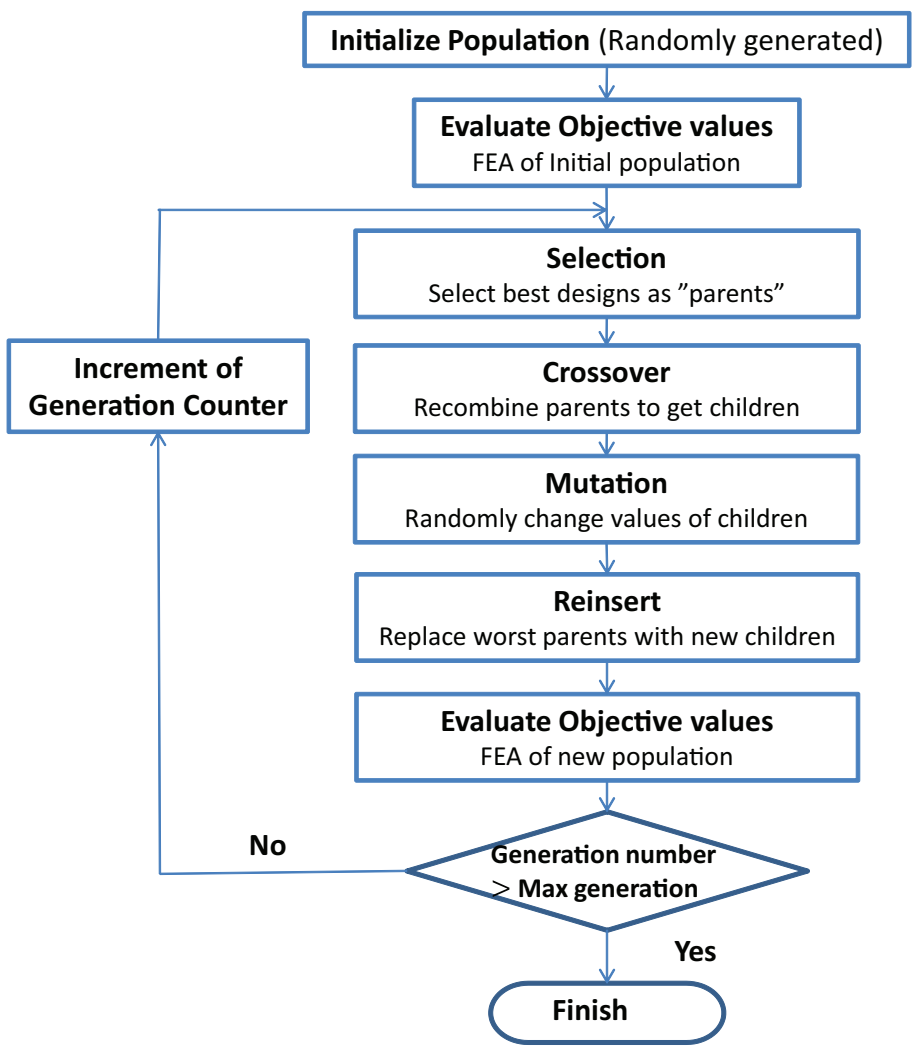

Figure 12: Flow chart of genetic algorithm.

\section{A. Objective and Fitness Functions}

The selection of individuals could be made on basis of the values of the objective function associated with each individual, however this approach is problematic as the objective may become negative (although not in the present example) and the range of objectives may be so large that individuals with a small objective dominate the reproduction resulting in rapid convergence to possibly local optima. A suitable mapping of the objective into a fitness function is necessary. ${ }^{19,22}$

Here a method proposed by Baker ${ }^{23}$ is used to avoid this issue. In Baker's method, the fitness of an individual is determined based on its rank in the population rather than the value of the objective function. Baker suggested that by limiting the range of relative fitness, no individuals can generate excessive offspring and so prevent premature convergence. The selective pressure or maximum range, MAX, is used to limit the range of relative fitness. Individuals are sorted in descending order and the fitness is calculated as: ${ }^{23}$

$$
F\left(x_{i}\right)=2-M A X+2(M A X-1) \frac{x_{i}-1}{N_{\text {ind }}-1}
$$

Here, $x_{i}$ is the position of the $i$-th individual and $N_{i n d}$ is the total number of individuals in the population. For example, the least fit individual which has the largest objective value and is ranked first has a fitness of 0 ; the best fit individual has the smallest objective value and has a fitness of $M A X$. Thus, the difference between the fitness of adjacent individuals can be limited to $\frac{2(M A X-1)}{N_{i n d}-1} ; M A X$ is typically chosen in the interval $[1.1,2] .{ }^{22}$ Here we choose $M A X=2$. 


\section{B. Selection}

Selection of suitable parents to evolve the next generation is the key part of the optimization process. ${ }^{24}$ Stochastic Universal Sampling (SUS), introduced by Baker, ${ }^{25}$ is used in this paper. SUS creates $N$ equally spaced pointers, where $N$ is the number of selections required. The population is shuffled randomly and a single random number, $r$, in the range $[0, S u m / N)$ is generated, where Sum is the sum of fitnesses of the individuals. The $N$ individuals are then chosen by generating $N$ pointers spaced by $S u m / N,[r, r+$ $\left.\frac{S u m}{N}, \ldots, r+\frac{S u m}{N}(N-1)\right]$, and selecting the individuals whose fitnesses span the positions of the pointers, as shown in Figure 13. In the above example, $N=4$ and $A$ to $G$ are the individuals. The four pointers are in the range of individuals $A, B, C$ and $F$, so these individuals are selected.

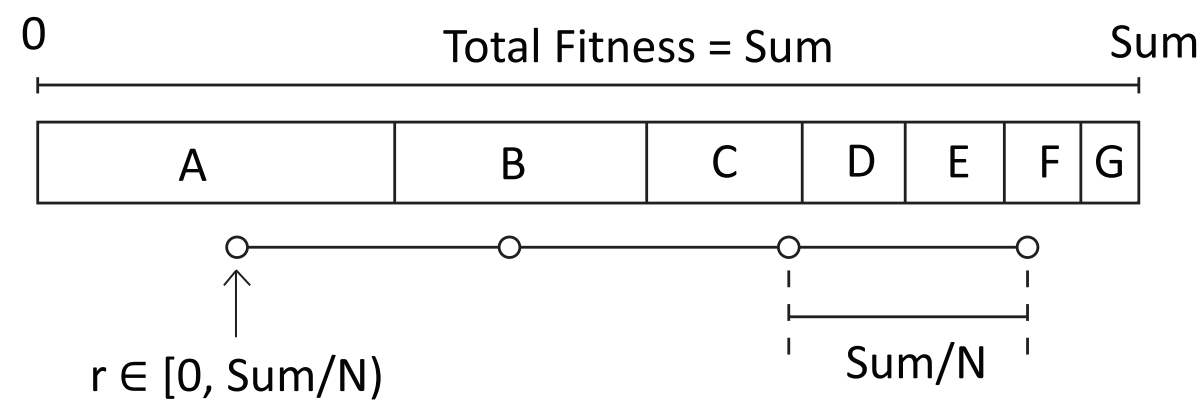

Figure 13: Schematic of stochastic universal sampling. ${ }^{26}$

\section{Crossover and Mutation}

Crossover produces new individuals that have some parts of both parents' genetic information. Each design variable is encoded into a 25-digit binary number, which is called a chromosome. Single-point crossover is used in this paper. An integer position, $i$, is selected randomly between 1 and the chromosome length, $l$, minus one $[1, l-1]$, and the genetic information at $[i, l]$ is exchanged between the two individuals. Figure 14 shows an example of crossover with $i=8$ and $l=10$. The binary numbers at $[8,9,10]$ of parents $P 1$ and $P 2$ are exchanged to obtain offspring $O 1$ and $O 2$.

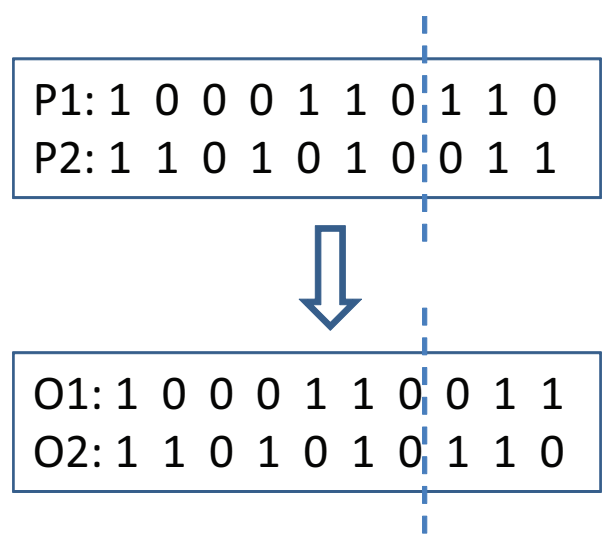

Figure 14: Crossover, $\mathrm{i}=8$.

Mutation is applied to increase the diversity of the population; some binary numbers of a chromosome are selected, based on the mutation probability and then flipped, as shown in Figure 15. The mutation probability is usually very low, typically in the range $0.001,0.01 .{ }^{22}$ We choose the mutation probability as 0.01 in this paper. After crossover and mutation, the next generation of individuals is produced and then inserted into the parents' generation to replace the least fit individuals. 


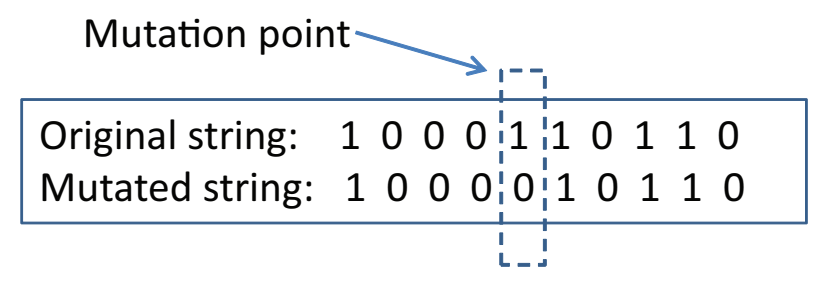

Figure 15: Mutation.

\section{Optimization Implementation}

We have developed a MATLAB script that connects topology generator, CAD software, FEA software and optimizer to implement the complete topology optimization cycle. Figure 16 shows the flow chart of this process. The Mesh generator, MESH2D, reads the structural size field as an input, computes the position of the nodes and their connectivity, and exports the topology through text files. Rhino 3D (Version 5 WIP), a NURBS-based CAD software, is adopted to create CAD files for finite element analysis. Rhino 3D can be run with Python scripts that parameterize a CAD model and automate the model generation. Next, the FEA software Abaqus/CAE software reads the files generated by Rhino 3D and evaluates the structural performance. Abaqus/CAE can also be run in batch mode with Python scripts that automate the finite element analysis. The mass of the structure, its deformation, maximum stress and critical buckling load are obtained at this point. Based on the computed structural performance, the optimization solver ${ }^{21}$ updates the three design variables. Rhino $3 \mathrm{D}$ reads the new variables and creates the next set of designs. The whole procedure is automated by our MATLAB script.

\section{Results}

In this section we present the results of the beam design problem. Two levels of optimization have been carried out, structural topology optimization and structural size optimization. The results of the beam design problem with microstructure of varying topology and fixed cross-sectional size are presented next. They are followed by the results of a size optimization problem that uses the topology obtained previously.

\section{A. Topology Optimization}

We have chosen Titanium alloy Ti-4AI-4V, with $\sigma_{Y}=970 \mathrm{MPa}, E=114 \mathrm{GPa}$ and $\rho=4430 \mathrm{~kg} / \mathrm{m}^{3}$, for the structure. The outer skin thickness, $t$, and the diameter of the rods, $d$, that form the microstructure have fixed values $t=d=2 \mathrm{~mm}$. Recall that the objective function is

$$
f(\vec{h})=\omega_{1} \frac{\operatorname{Mass}(\vec{h})}{\operatorname{Mass}_{0}}+\omega_{2} \frac{U(\vec{h})}{U_{0}}, \vec{h}=\left(h_{1}, h_{2}, \ldots, h_{36}\right)
$$

Here we take $\omega_{1}=0.3$ and $\omega_{1}=0.7$. The reference values, Mass $_{0}$ and $U_{0}$, are the mass and strain energy of the structure with $h_{1}=h_{2}=\ldots=h_{36}=2 h_{0}$, where $h_{0}=0.0064 \mathrm{~m}$, Mass $_{0}=0.3086 \mathrm{~kg}$ and $U_{0}=1.2431 \mathrm{Nm}$. The reference structure is shown in Figure 17. There are 36 nodes in the background mesh (Figure 10). We set the values of $h_{i}$ on nodes with the same Y-coordinate to be equal and hence the number of design variables is reduced to 6 :

$$
\vec{h}=\left(h_{1}, h_{2}, \ldots, h_{6}\right)
$$

There are 20 individuals in each generation and after running the analysis for 100 generations it converges to a minimum. The optimized structural size field and corresponding structure are shown in Figure 18 . It can be seen that the microstructure is much finer at top and bottom than in the middle. I-beams are known to be an efficient shape; the beam obtained here is similar to an I-beam and so this result matches our expectation. The evolution of objective function, mass, strain energy, and constraints are shown in Figures 19, 20 and 21. The final values of the objective, mass and strain energy are $0.8972,0.4249 \mathrm{~kg}$ and 0.8598 $\mathrm{Nm}$, respectively. 


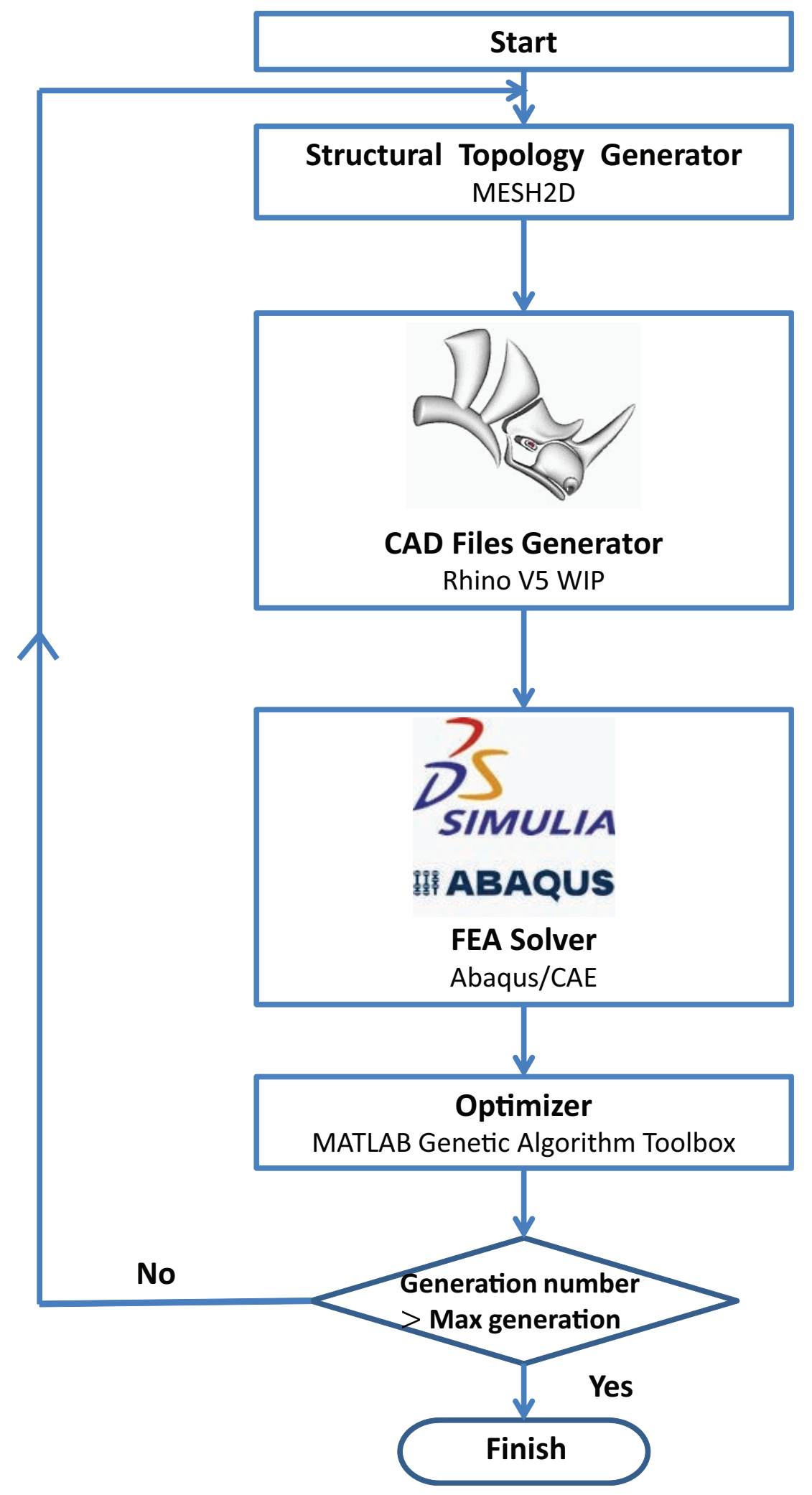

Figure 16: Flow chart of optimization procedure. 


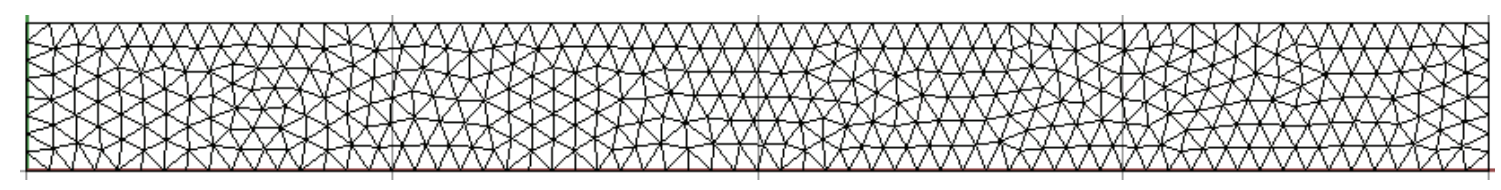

Figure 17: Reference structure with uniform size field: $h_{1}=h_{2}=\ldots=h_{6}=0.0128 \mathrm{~m}$

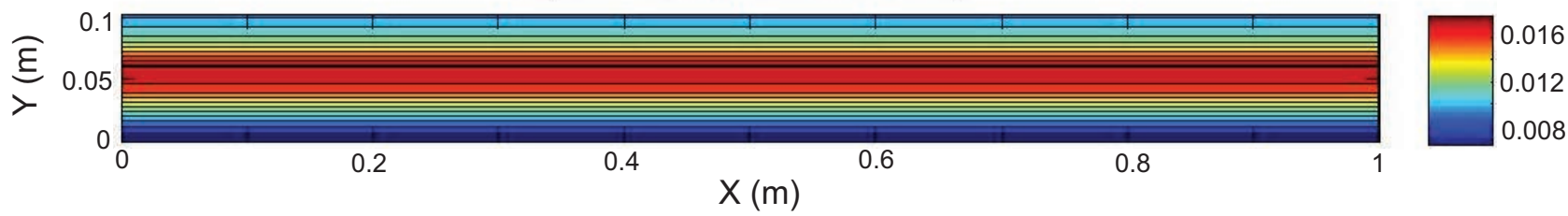

(a)

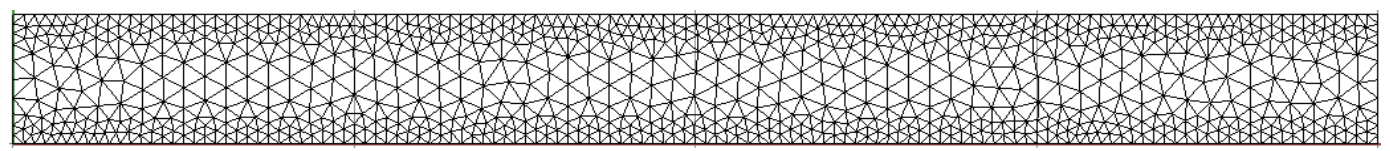

(b)

Figure 18: Optimized structural size field and topology.

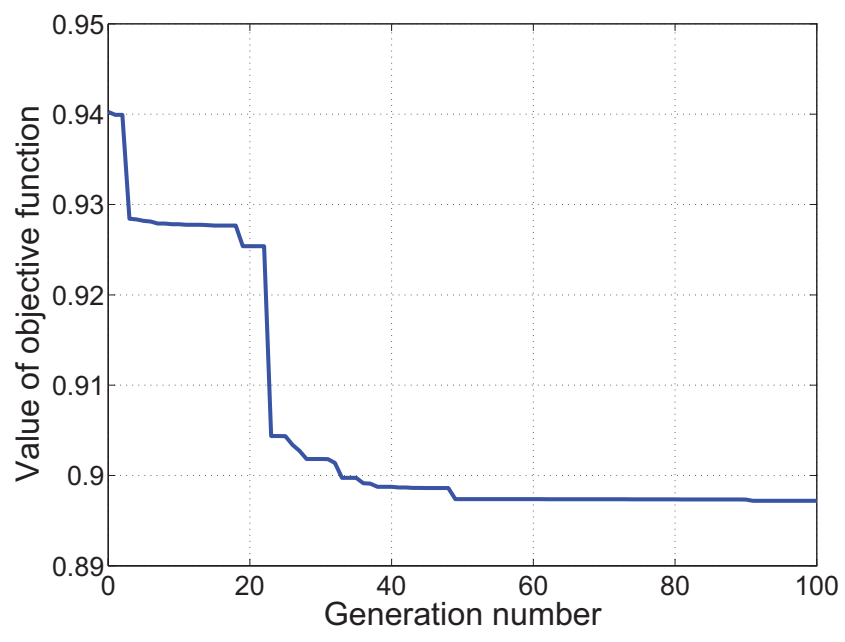

(a)

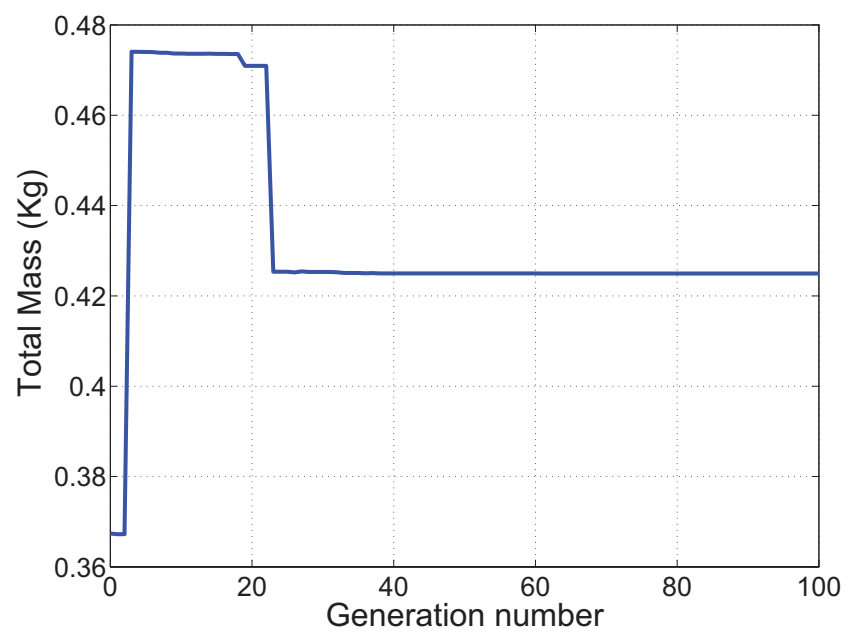

(b)

Figure 19: Evolution of solutions: (a) value of objective function; (b) mass. 


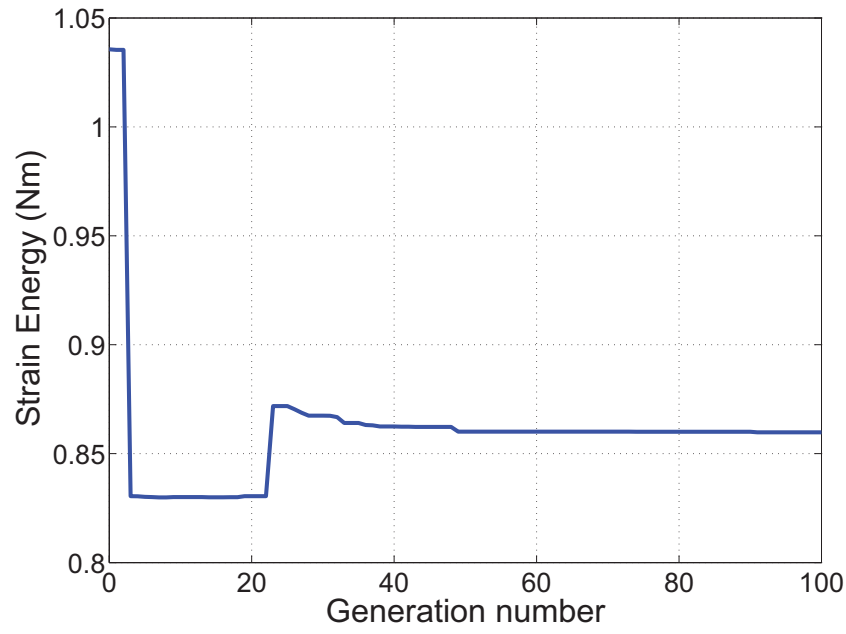

(a)

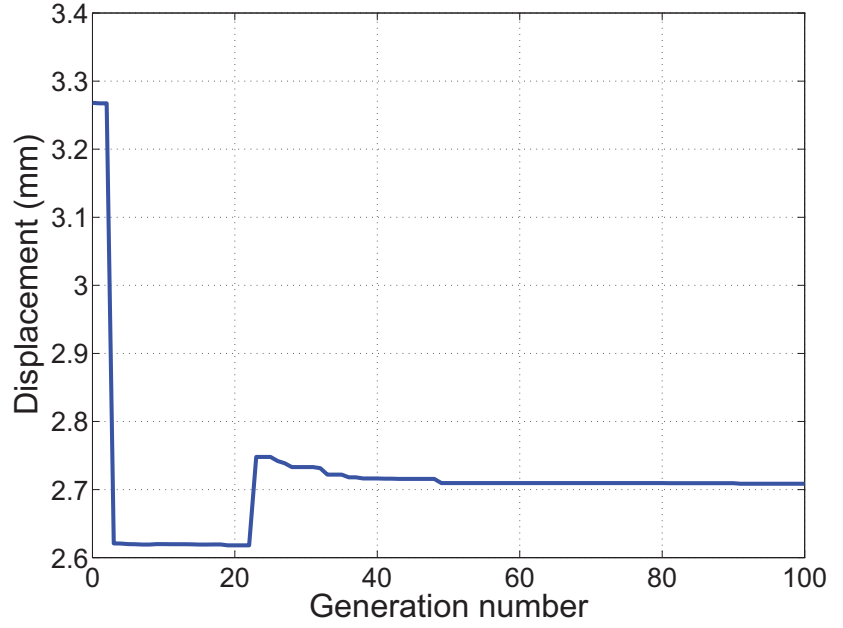

(b)

Figure 20: Evolution of solutions: (a) strain energy; (b) maximum deflection of top surface of beam.

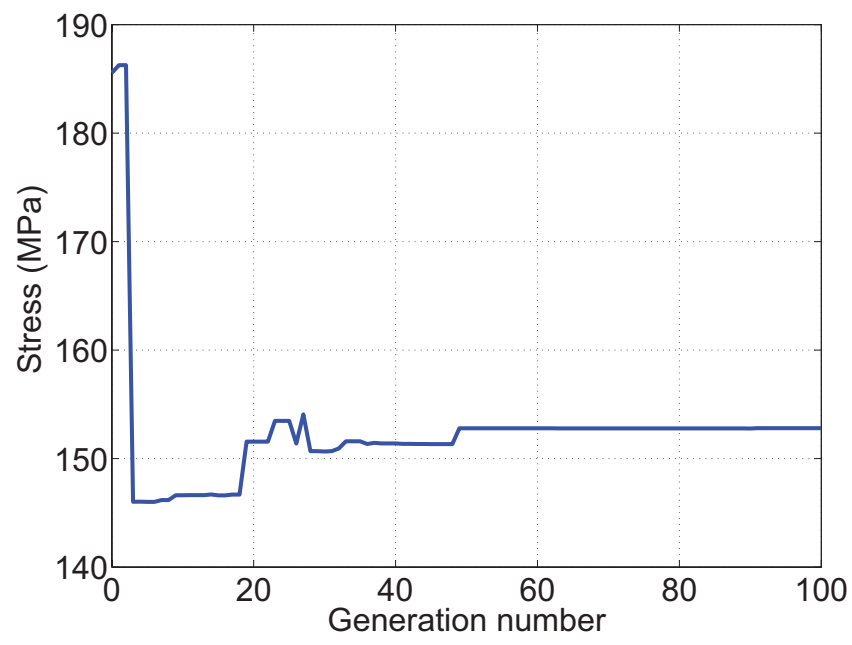

(a)

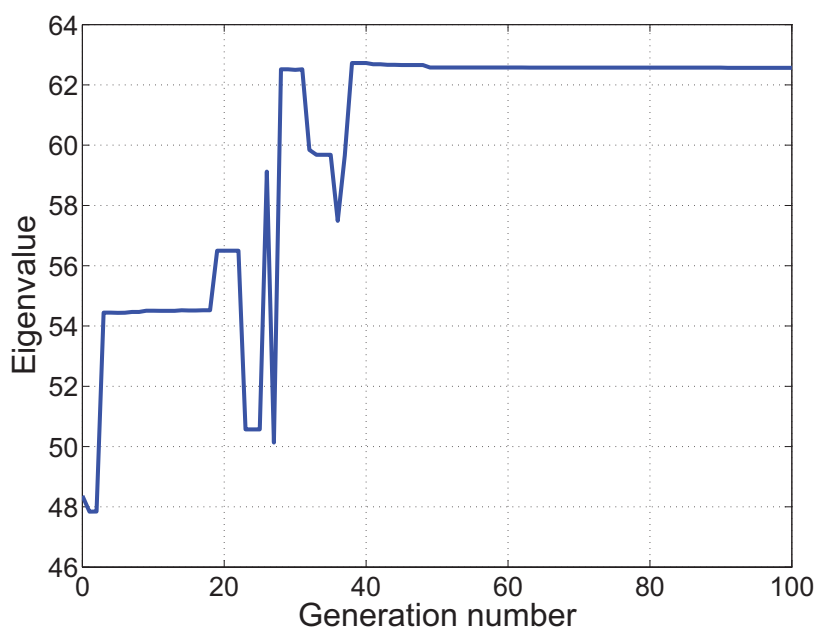

(b)

Figure 21: Evolution of solutions: (a) maximum Mises stress; (b) eigenvalue $\lambda$. 


\section{B. Size Optimization}

In the previous section, the thickness of the outer skin and the rods were both fixed at $2 \mathrm{~mm}$. In this section we choose the topology obtained in the previous section and optimize $t$ and $d$. The size optimization is formulated as follows:

$$
\begin{array}{cc}
\text { Minimize : } & f(t, d)=\omega_{1} \frac{\operatorname{Mass}_{(t, d)}}{\operatorname{Mass}_{0}}+\omega_{2} \frac{U(t, d)}{U_{0}} \\
\text { subject to : } \sigma_{Y} \leq \sigma_{0} & \text { (Stress constraint) } \\
\delta \leq 4 \mathrm{~mm} & \text { (Deformation constraint) } \\
\lambda \geq 1 & \text { (Buckling constraint) } \\
\text { Fixed topology } & \text { (Topology constraint) } \\
1 \mathrm{~mm} \leq t \leq 5 \mathrm{~mm} & \text { (Size constraint 1) } \\
1 \mathrm{~mm} \leq d \leq 4 \mathrm{~mm} & \text { (Size constraint 2) }
\end{array}
$$

The lower limit, $1 \mathrm{~mm}$, is set by Electron Beam Melting technology; the upper limit on $d$ is set to avoid that adjacent members of microstructure become merged. Similarly, in order to avoid overlap between outer skin and its adjacent microstructure, the upper limit of $t$ is also set. The upper limits have been calculated by considering a microstructure with minimum edge length of $6.4 \mathrm{~mm}$ which is the smallest value of the structural size field $h 0$ as considered in Equation 5.

The evolution of $t, d$, objective function, mass, strain energy, and constraints are shown in Figures 22, 23, 24 and 25. The final diameter of the rods and the thickness of the skin are $1 \mathrm{~mm}$ and $4.4 \mathrm{~mm}$, respectively. The final value of the objective has been reduced to 0.5574 . The mass and strain energy are $0.2886 \mathrm{~kg}$ and $0.4917 \mathrm{Nm}$, respectively. These results will be discussed in the next section.

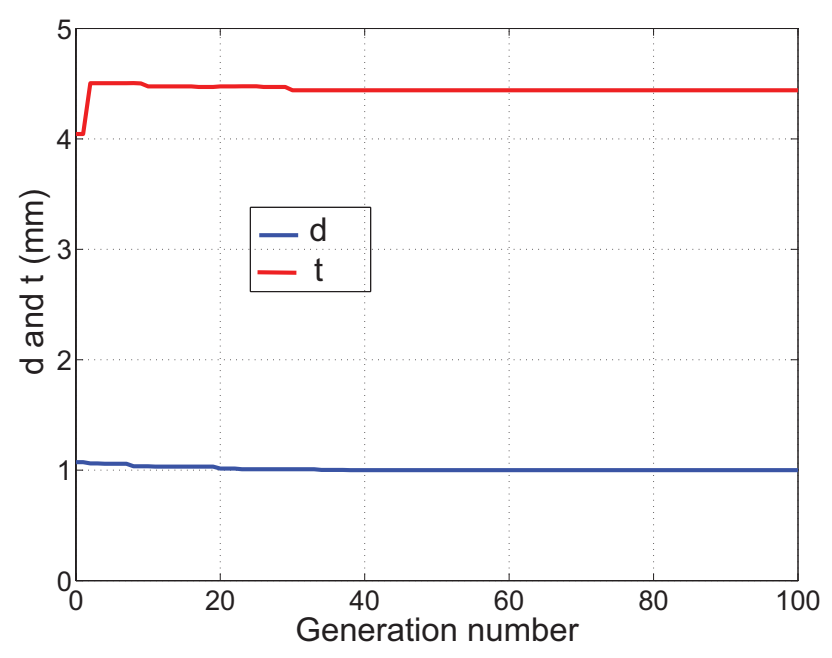

Figure 22: Evolution of diameter of microstructure, $d$, and thickness of skin, $t$.

\section{Comparison with Conventional Beams}

In this section we compare the results obtained from implicit topology optimization to conventionally designed beams. We will first introduce two dimensionless metrics to evaluate beam performance, and a shape parameter that characterizes a solid beam cross-section. Then the performance of solid beams and a simple truss structure is compared with solutions obtained from the optimization.

\section{A. Performance Metrics}

The performance of a simply supported beam with uniform cross-section and loaded by uniformly distributed load is governed by six parameters: mass $m$, density $\rho$, beam length $l$, total external force $F$, Young's modulus 


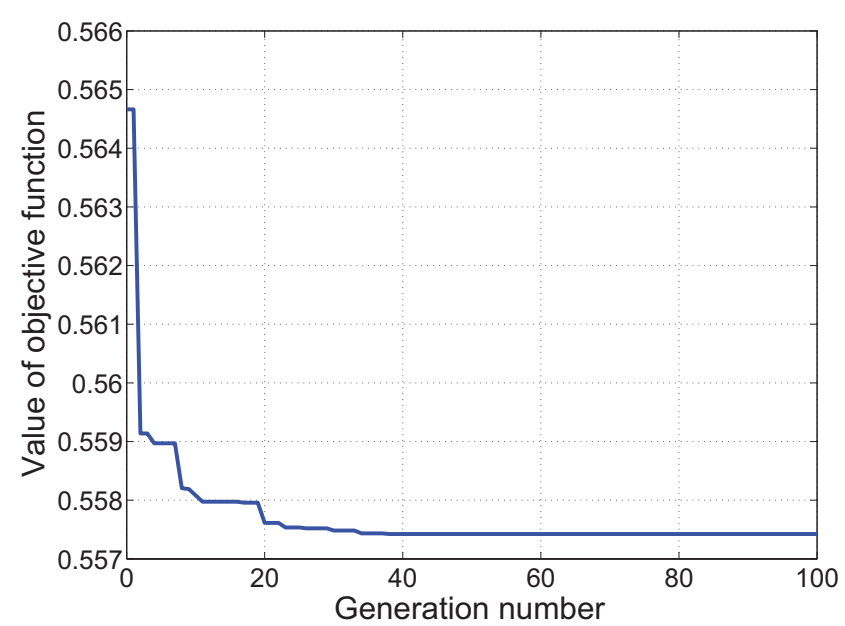

(a)

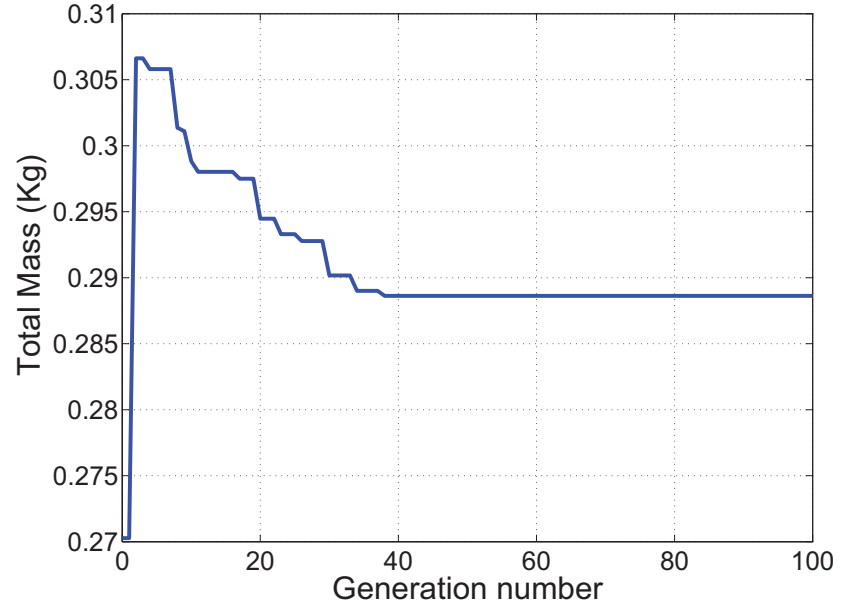

(b)

Figure 23: Evolution of solutions: (a) value of objective function; (b) mass.

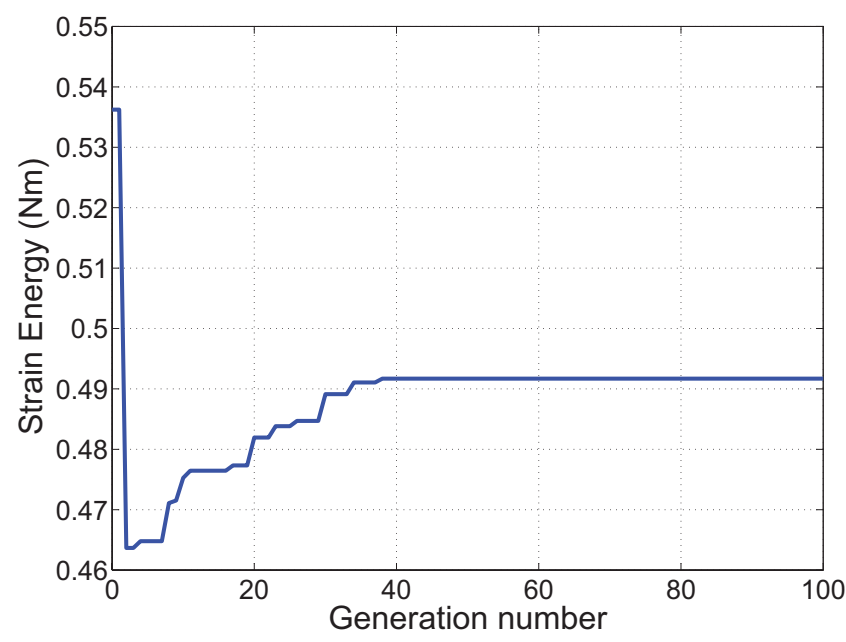

(a)

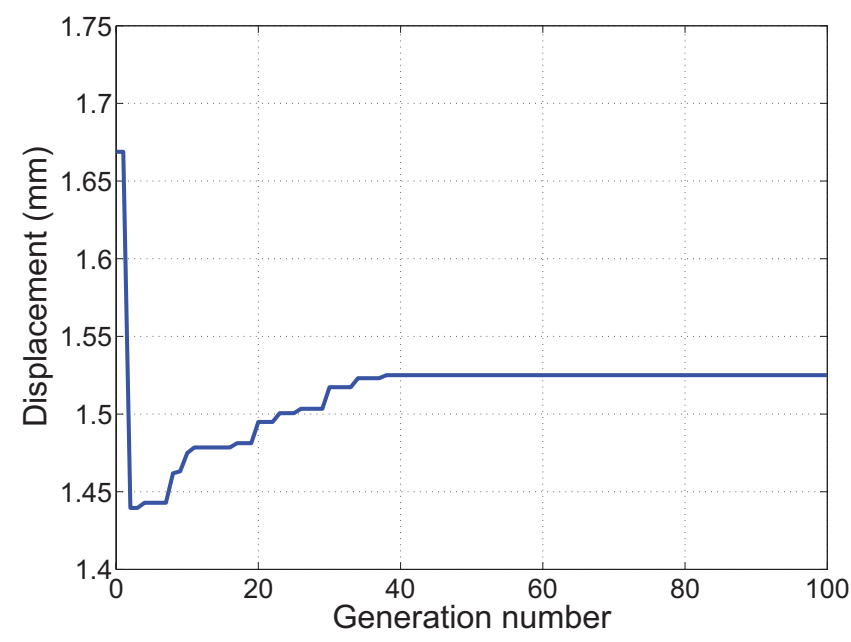

(b)

Figure 24: Evolution of solutions: (a) strain energy; (b) maximum deflection of top surface of beam. 


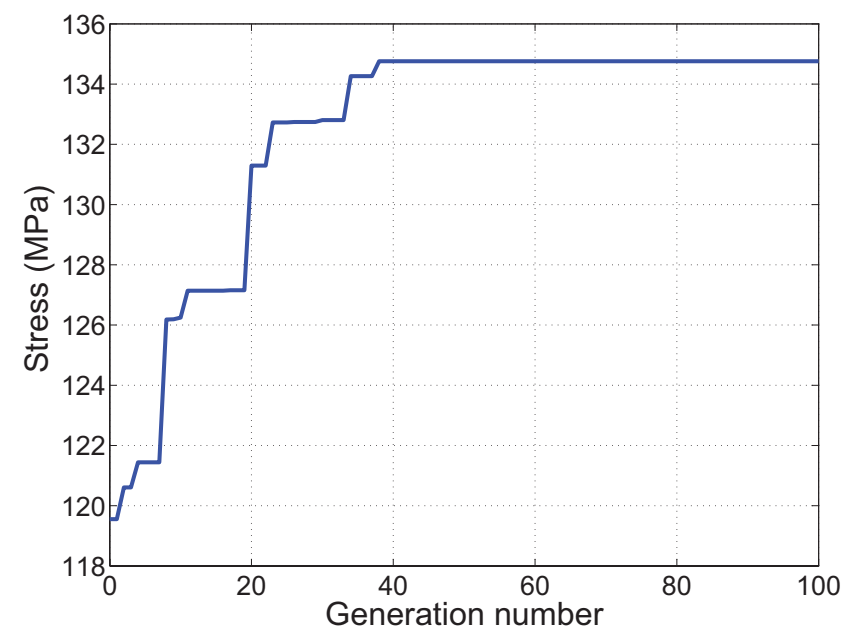

(a)

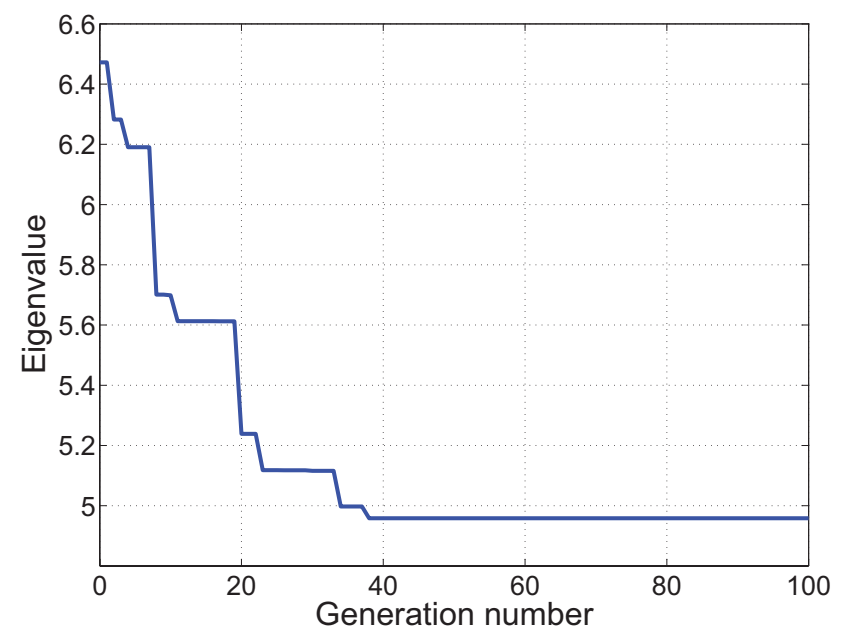

(b)

Figure 25: Evolution of solutions: (a) maximum Mises stress; (b) eigenvalue $\lambda$.

$E$, and maximum deflection $\delta$. The dimensions of these quantities can be expressed as a product of the basic physical dimensions mass, length and time, represented by $\mathbf{M}, \mathbf{L}$ and $\mathbf{T}$, respectively. Their dimensions are listed in Table 1.

Table 1: Summary of parameters' dimensions

\begin{tabular}{lc}
\hline \hline Quantity & Dimension \\
\hline Mass, $m$ & $\mathbf{M}$ \\
Density, $\rho$ & $\mathbf{M L}^{-\mathbf{3}}$ \\
Beam length, $l$ & $\mathbf{L}$ \\
Total external force, $F$ & $\mathbf{M L T}$ \\
Young's Modulus,$E$ & $\mathbf{M L}^{-\mathbf{1}} \mathbf{T}^{-\mathbf{2}}$ \\
Deformation, $\delta$ & $\mathbf{L}$ \\
\hline \hline
\end{tabular}

Since the quantity $\rho l^{3}$ has dimension of $\mathbf{M}$, a dimensionless mass parameter is $m / \rho l^{3}$. The stiffness $F / \delta$ has dimensions of $\mathbf{M T}^{-2}$ and the combination of the Young's modulus and beam length, $E l$, also has dimensions of $\mathbf{M} \mathbf{T}^{-\mathbf{2}}$. Hence a dimensionless stiffness can be defined as $F / E l \delta$. These two dimensionless parameters, $m / \rho l^{3}$ and $F / E l \delta$, are adopted to evaluate the structural performance.

\section{B. Comparison with Solid Beam}

\section{Shape Factor for Elastic Bending}

The shape of a beam's cross-section significantly affects its efficiency. Beams with hollow-box or I-section are better than solid square sections of the same cross-sectional area. In order to characterize this, we use the shape factor $\phi$, introduced by Ashby. ${ }^{27}$ This factor measures the structural efficiency of a section shape and is independent of material properties. The shape factor $\phi$ is a ratio between the stiffness of the section of interest and a reference shape, which is taken to be a solid square section with the same cross-sectional area. The bending stiffness $S$ of a beam is proportional to $E I$, where $I$ is the second moment of area.

$$
S \propto E I
$$

The second moment of area, $I_{0}$, for the reference beam of square section, with edge length $b_{0}$ and area 
$A=b_{0}^{2}$ is

$$
I_{0}=\frac{b_{0}^{4}}{12}=\frac{A^{2}}{12}
$$

The shape factor for elastic bending is derived as:

$$
\phi=\frac{S}{S_{0}}=\frac{E I}{E I_{0}}=\frac{12 I}{A^{2}}
$$

The shape factor for elastic bending is a dimensionless parameter that only depends on shape; beams of any scale have the same value of $\phi$ if their section shapes are the same. ${ }^{27}$

For a beam with rectangular cross-section, Figure 26, the shape factor can be calculated as:

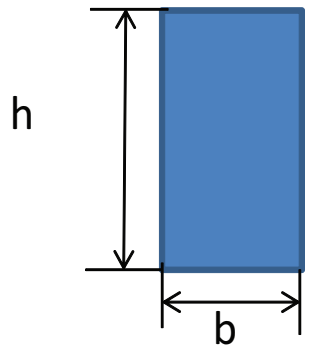

Figure 26: Cross-section with rectangular shape

$$
\phi=\frac{12 I}{A^{2}}=\frac{b h^{3}}{b^{2} h^{2}}=\frac{h}{b}
$$

and from this equation it follows that high values of $\phi$ are obtained by increasing the aspect ratio $\frac{h}{b}$. If the section of a beam is elongated, or hollow, or I-shaped, or a thin-walled tube, then $\phi$ can have values of 50 or even larger. ${ }^{27}$ However, due to the manufacturing and material constraints, large values of $\phi$ may not be feasible. Assuming beams with high $\phi$ could be fabricated, the upper bound of $\phi$ is set by material properties. Analyses done by Gerard, ${ }^{28}$ Weaver and Ashby ${ }^{29}$ indicate that the maximum practical shape efficiency is limited by local buckling. Ashby ${ }^{27}$ has estimated the following upper bound for $\phi$ :

$$
\phi_{\text {max }} \approx 2.3 \sqrt{\frac{E}{\sigma_{f}}}
$$

where $\sigma_{f}$ is the material failure stress. For beams made of Titanium alloy Ti- $4 \mathrm{AI}-4 \mathrm{~V}$, we take $\sigma_{f}$ as the yield strength $\sigma_{f}=970 \mathrm{MPa}$ and obtain $\phi_{\max }=25$.

\section{Performance Comparison}

The two performance metrics and the shape factor can be combined to obtain a relation between section shape and performance. The analytical solution for the problem that is being considered is:

$$
\delta=\frac{5 F l^{3}}{384 E I}
$$

Hence the second moment of area can be written as:

$$
I=\frac{5 F l^{3}}{384 E \delta}
$$

Substituting the expression for the shape factor, Equation 12, into Equation 16 we obtain:

$$
A^{2}=\frac{15 F l^{3}}{96 E \delta \phi}
$$


Substituting $m=\rho A l$ and after some algebra, we obtain:

$$
\left(\frac{m}{\rho l^{3}}\right)^{2}=\frac{F}{E l \delta} \frac{15}{96 \phi}
$$

Figure 27 shows a plot of $m / \rho l^{3}$ versus $F / E l \delta$ for $\phi=1, \phi=5, \phi=10, \phi=55.1$ and $\phi=212.1$ for solid beams. Two additional points mark the structures obtained in Section VI. The figure shows that the two solutions from optimization have equivalent performance to beams with $\phi=55.1$ and $\phi=212.1$. Recalling that the maximum value of the shape factor $\phi$ for solid beams is approximately 25 , it can be concluded that the structures obtained by implicit topology optimization are much better than the best possible solid beams.

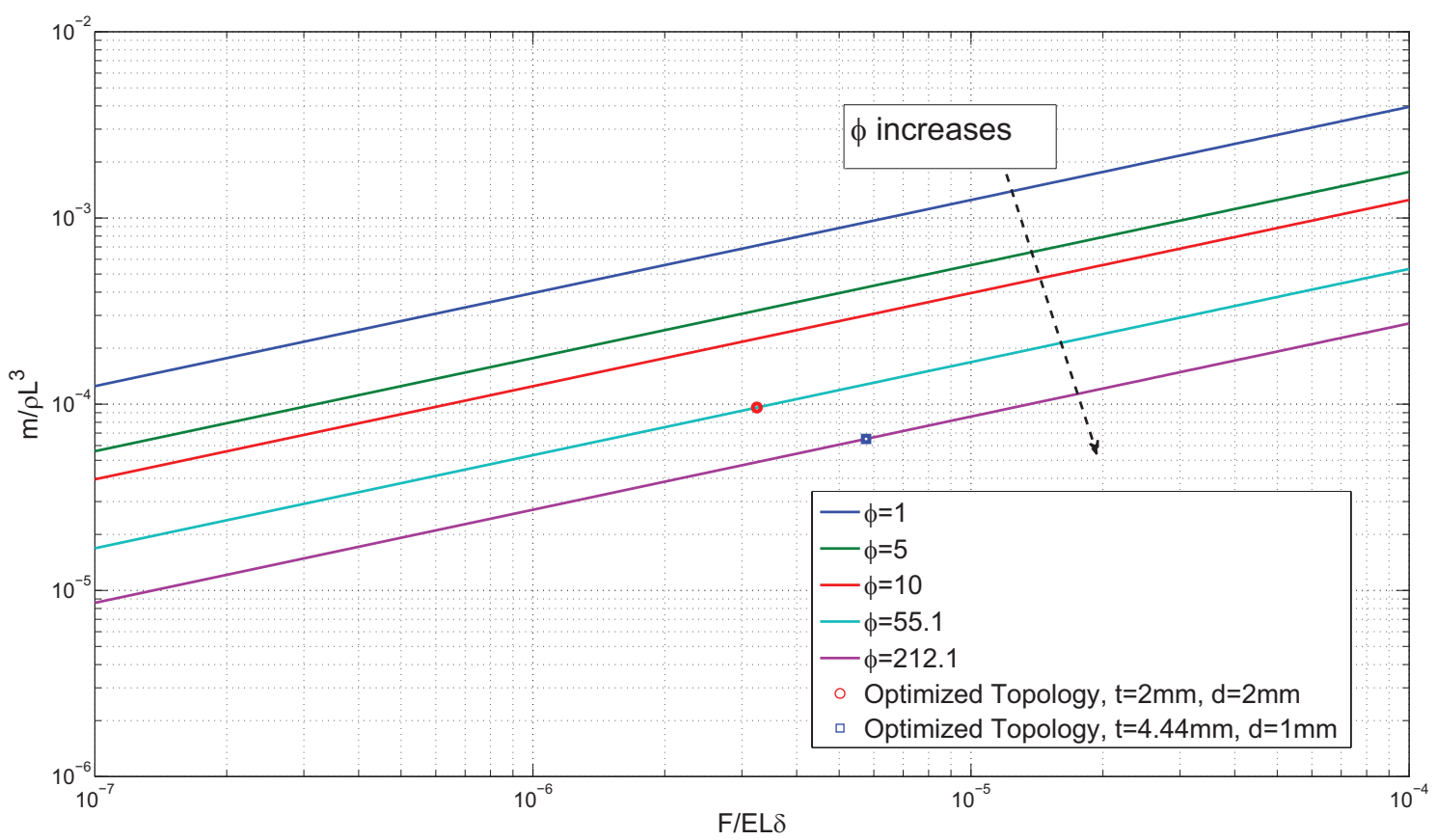

Figure 27: Performance comparison with solid beams.

\section{Comparison with Simple Truss}

The simple truss shown in Figure 28 is compared with the beams obtained by optimization. The two structures with optimized microstructure had $t=2.0 \mathrm{~mm}$ and $d=2.0 \mathrm{~mm}$ when only the topology was optimized, and $t=4.44 \mathrm{~mm}$ and $d=1.0 \mathrm{~mm}$ when the cross-sections were also optimized. Keeping both the topology and the ratio $\frac{t}{d}$ fixed but varying the scale, we have obtained the performance curves for the cases $\frac{t}{d}=\frac{2}{2}$ and $\frac{t}{d}=\frac{4.44}{1}$. These curves are plotted in Figure 29.

We have also optimized the member cross-sections of the truss. Assuming the upper and lower chords to have square cross-section of size $t$ and the diagonal to be circular rods with diameter $d$ we obtained $t=4.62 \mathrm{~mm}$ and $d=3.34 \mathrm{~mm}$. The performance of two truss designs, one with the ratio $\frac{t}{d}=\frac{2}{2}$ and the other with the optimized ratio $\frac{t}{d}=\frac{4.62}{3.34}$, are also plotted in Figure 29. This figure shows that the best optimized beam lies just below the best truss.

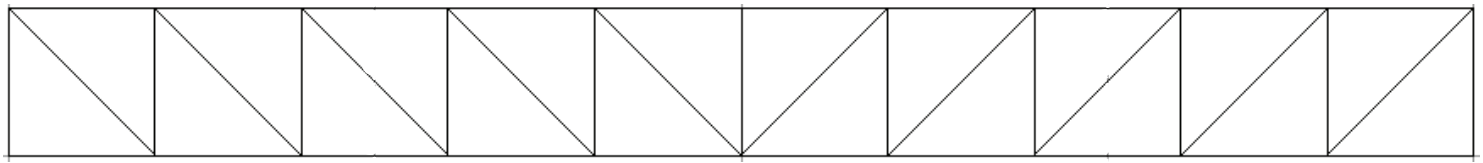

Figure 28: Simple symmetric truss. 


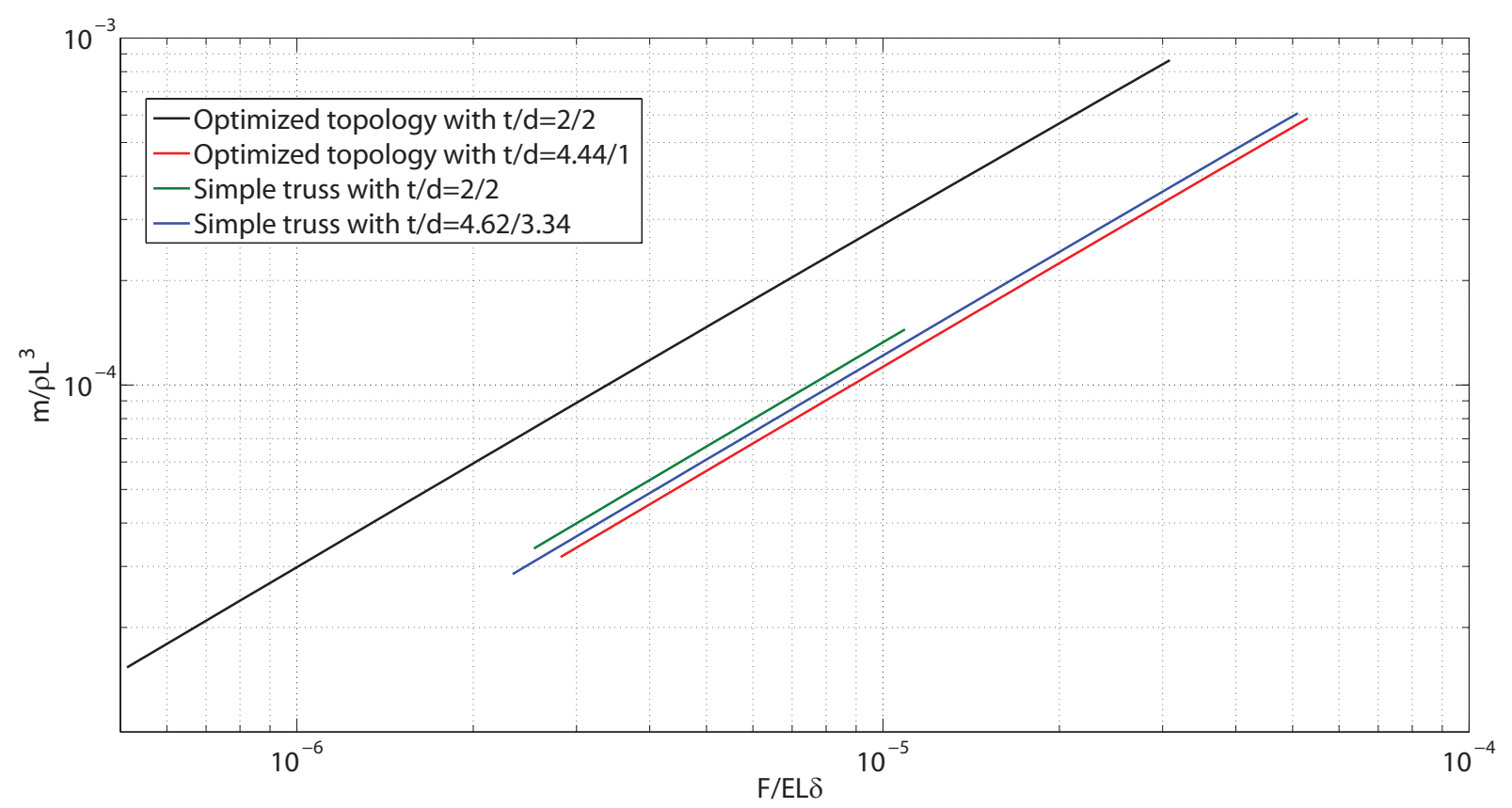

Figure 29: Performance comparison with simple truss.

\section{Conclusion}

The objective of this study was to introduce an implicit topology optimization method for designing and manufacturing lightweight structural components. The implicit topology optimization method developed in this paper, based on varying the structural size field by a genetic algorithm, has been shown to be a way to avoid impractical structures or a huge design space. By studying a simple and yet classical beam design problem, we have obtained optimized beams with a denser microstructure near the faces, which satisfies our intuition that optimized structures should be similar to an I-beam.

Two levels of optimization, topology optimization and size optimization using a previously optimized topology, were studied in this paper. Comparing with the initial reference structure, the objective function has been reduced by $10.3 \%$ by the topology optimization and $44.3 \%$ by the size optimization. A detailed implementation of the optimization loop using commercial CAD and FEA software, running under a genetic algorithm has been developed. It has been found that using a MATLAB script as an interface to connect these software packages is efficient, robust, and quite general.

Two performance metrics were introduced and the optimized beams obtained in the present study were compared with solid beams and a simple truss. Our comparison has shown that the optimized structures are superior to these other structures.

\section{Acknowledgements}

We thank Ted Nye for inspiring this research and gratefully acknowledge the financial support received from Northrop Grumman Aerospace Systems.

\section{References}

${ }^{1}$ K. Saitou, K. Izui, S. Nishiwaki, and P. Papalambros, "A survey of structural optimization in mechanical product development", Journal of Computing and Information Science in Engineering, Vol. 5, 2005, pp. 214-226.

${ }^{2}$ M. P. Bendsøe and N. Kikuchi, "Generating Optimal Topologies in Structural Design Using a Homogenization Method",Computer Methods in Applied Mechanics and Engineering, Vol. 71, 1988, pp. 197-224.

${ }^{3}$ M. P. Bendsøe, "Optimal Shape Design as a Material Distribution Problem," Struct. Optim., Vol. 1, ,1989, pp. 193-202.

${ }^{4}$ M. P. Bendsøe and O. Sigmund, "Topology Optimization theory, methods, and applications", Springer, Berlin, Gremany, 2003, ISBN-3540429921. 
${ }^{5}$ M. Santer and S. Pellegrino, "Topological Optimization of Compliant Adaptive Wing Structure", AIAA Journal, Vol. 47, 2009, pp. 523-534.

${ }^{6}$ P. Winslow, "Synthesis and Optimization of Free-form Grid Structures", Ph.D thesis, Department of Engineering, University of Cambridge, 2009.

${ }^{7}$ Lu, K.-J. and Kota, S., "An Effective Method of Synthesizing Compliant Adaptive Structures using Load Path Representation", Journal of Intelligent Material Systems and Structures, Vol. 16, 2004, pp. 307-317.

${ }^{8} \mathrm{Lu}, \mathrm{K} .-\mathrm{J}$. and Kota, S., "Topology and Dimensional Synthesis of Compliant Mechanisms using Discrete Optimization", Journal of Mechanical Design, Vol. 128, 2006, pp. 1080-1091.

${ }^{9}$ G. M. Reddy and J. Cagan, "Optimally Directed Truss Topology Generation Using Shape Annealing", Journal of Mechanical Design, Vol. 117, 1995, pp. 206-210.

${ }^{10} \mathrm{~K}$. Shea and J. Cagan, "Innovative dome design: Applying geodesic patterns with shape annealing", Artificial intelligence for engineering design, analysis and manufacturing, Vol. 11, 1997, pp. 379-394 .

${ }^{11}$ M. Agarwala, D. Bourell, J. Beaman, H. Marcus and J. Barlow, "Direct selective laser sintering of metals", Rapid Prototyping Journal, Vol. 1 Iss: 1, pp.26 - 36, 1995.

${ }^{12}$ J-P. Kruth, P. Mercelis, J. Van Vaerenbergh, L. Froyen, and M. Rombouts "Binding mechanisms in selective laser sintering and selective laser melting", Rapid Prototyping Journal, Vol. 11 Iss: 1, pp.26 - 36, 2005. 2008.

${ }^{13} \mathrm{~J}$. Wooten and D. P. Dennies, "Electron Beam Melting Manufacturing for Production Hardware", SAE International,

${ }^{14}$ K. Deb, "Multi-objective optimization using evolutionary algorithms", John Wiley \& Sons, Chichester, England, 2003, ISBN-978-0471873396.

${ }^{15}$ P.-O. Persson, G. Strang, "A Simple Mesh Generator in MATLAB", SIAM Review, Vol. 46 (2), 2004, pp. 329-345.

${ }^{16}$ P. J. Frey and P.-L. George, "Mesh Generation, Application to Finite Elements",John Wiley Sons, Chichester, UK, 2008, ISBN-978-1903398005.

${ }^{17}$ P.-L. George, H. Borouchaki, P. J. Frey, P. Laug and E. Saltel "Mesh Generation and Mesh Adaptivity: Theory and Techniques", Encyclopdedia of Computational Mechanics,John Wiley Sons, Chichester, UK, 2007, ISBN-0-470-84699-2.

${ }^{18}$ D. Engwirda, "MESH2D - Automatic Mesh Generation", http://www.mathworks.com/matlabcentral/fileexchange/25555mesh2d-automatic-mesh-generation

${ }^{19}$ D. Goldberg, "Genetic Algorithms in Search, Optimization, and Machine Learning", Addison-Wesley Professional, 1989, ISBN-0201157675.

${ }^{20}$ K. Rasheed, H. Hirsh, and A. Gelsey, "A Genetic Algorithm for Continuous Design Space Search", Artificial Intelligence in Engineering, Vol. 11, No. 3,1997, pp. 295-305.

${ }^{21}$ A. J. Chipperfield and P. J. Fleming, "The MATLAB Genetic Algorithm Toolbox", Department of Automatic Control and Systems Engineering of The University of Sheffield, UK.

${ }^{22}$ A. J. Chipperfield, P. J. Fleming, H. Pohlheim and C. Fonseca, "The MATLAB Genetic Algorithm Toolbox, Users' Guide", Department of Automatic Control and Systems Engineering, University of Sheffield, UK.

${ }^{23}$ J. E. Baker, "Adaptive Selection Methods for Genetic Algorithms", Proc. ICGA 1, pp. 101-111, 1985.

${ }^{24}$ P. Winslow, S. Pellegrino and S. B. Sharma "Multi-objective Optimization of Free-form Grid Structures", Struct Multidisc Optim, Vol. 40, 2010, pp. 257-269.

${ }^{25}$ J. E. Baker, "Adaptive Selection Methods for Genetic Algorithms", Proc. ICGA 2, pp. 14-21, 1987.

${ }^{26}$ Wikipedia, "Stochastic universal sampling", http://en.wikipedia.org/wiki/Stochastic_universal_sampling

${ }^{27}$ M. Ashby, "Material Selection in Mechanical Design", Butterworth-Heinemann, Oxford, UK, 2005, ISBN-0750661682.

${ }^{28}$ G. Gerard, "Minimum Weight Analysis of Compression Structures", New York University Press, New York, USA, 1956, Library of Congress Cataglog Number: 55-10052.

${ }^{29}$ P. M. Weaver and M. Ashby, "Material limits for shape efficiency", Progress in Materials Science, vol. 41, no. 12, pp. $61-128,1998$ 